\title{
POLYFIT ASSISTED MONOSCOPIC MULTI-IMAGE MEASUREMENT SYSTEMS
}

\author{
D. Suwardhi ${ }^{1,}$, S.W. Trisyanti ${ }^{1}$, L. Kamal ${ }^{1}$, H.A. Permana ${ }^{1}$, A. Murtiyoso ${ }^{2}$, K.N. Fauzan ${ }^{1}$ \\ 1 3D Modelling and Information System, Remote Sensing and GIS Group, Bandung Institute of Technology, Indonesia - \\ deni@gd.itb.ac.id, shafarina.wahyu@ students.itb.ac.id \\ ${ }^{2}$ Photogrammetry and Geomatics Group, ICube Laboratory UMR 7357, INSA Strasbourg, France - arnadi.murtiyoso@insa- \\ strasbourg.fr
}

Commission II

KEY WORDS: Photogrammetry, 3D Modeling, Semi-Automatic, Polygon-model, Point Cloud

\begin{abstract}
:
Nowadays, in light of the latest development in three-dimensional (3D) modeling technology, an essential role is given to the research and development of fully-automated or semi-automated processes in order to increase workflow effectiveness. A key challenge is thus to automate the process leading to the geometric model which supports the Building Information Modeling (BIM) or 3D-Geographical Information Systems (3D-GIS). This 3D model usually originates from image-based or range-based point clouds. This research is the beginning of the development of a 3D modeling approach that is semi-automatic, and possibly fullyautomatic, by combining polygon surface fitting (polyfit) technique and monoscopic multi-image measurement system. With the advent of dense matching and Structure from Motion methods (SfM), point clouds can be generated from multiple images obtained from digital cameras. Then, to reduce the data and to allow for efficient processing, it is necessary to extract polygonal surface data from point clouds delivered by the dense matching process. The polygonal surface is then used for the basis of further manual monoscopic measurements which are achieved separately on each image to obtain more detailed 3D model. Next, this approach analyzed the polygonal surface deformations in comparison to the initial point cloud data. It can be seen how the resolution and noise of the original point clouds affect the subsequent Polyfit-based modeling and monoscopic measurements. The deformations and the accuracy evaluation have been undertaken using different open source software. Also, the geometric error in the polyfitderived polyhedral reconstruction propagating to the subsequent monoscopic-derived measurements was evaluated. Finally, our modeling approach shows that it can improve the processing speed and level of detail of the 3D models achieved using existing monoscopic measurements. Typically geometric accuracy itself doesn't have enough information to make accurate geometry model.
\end{abstract}

\section{INTRODUCTION}

\subsection{Background}

According to Article 37 Paragraph (3) of the Indonesian Law No. 28 of 2002, concerning building construction, the preservation, maintenance, and inspection of buildings must be carried out in order to meet the requirements of functional worthiness. One of the activities to maintain and inspect buildings can be done by means of documentation. However, at present, documents in the form of classical drawings and maps are being replaced by three-dimensional (3D) models, while traditional measuring techniques are enhanced by range-based and image-based sensors.

One of the image-based measurement techniques is photogrammetric multi-image system that is designed to handle two or more overlapping photographs taken from different angles of an object to generate the 3D model. Usually, manual monoscopic measurements are achieved separately on each image to obtain three-dimensional model of an architectural or heritage objet. These systems don't give the opportunity of conventional stereo-photogrammetry (Hanke and Grussenmeyer, 2002). Unfortunately, manual measurement processes are time consuming and cost critical, especially in buildings with complex geometries. The next really important problem in relation to manual measurements is human error, whether it is positional errors of the digitizing, topological errors, or semantic attribution errors. Using automation will reduce the possibility of error even more so in a fast developing world where decisions are needed to be faster and more accurate. This necessitates the introduction of automatic digitization in 3D modeling. Automatic or semi-automatic digitization performs the same task as manual process but with minimum and/or no human interference. These techniques can be developed for all possible features.

With the advent of Semi-Global Matching (SGM) and Structure from Motion methods (SfM), dense point clouds can be made from images obtained from digital cameras. The quality of dense point clouds created from images using SGM are predominately affected by the ground sample distance (GSD) and image quality. In both 3D Geographical Information Systems (3D-GIS) and Building Information Modeling (BIM), 3D models are needed in vector geometry form. This means that the dense point cloud needs to be converted into geometrical primitives such as polygons. There are a lot of factors that will dictate such an undertaking; detail level, available equipment, point cloud processing and CAD software, to name a few.

PolyFit is an open source algorithm for polygonal surface reconstruction from point clouds. The current implementation exploits planar primitives and it is suitable for reconstructing

\footnotetext{
* Corresponding author
} 
piecewise planar objects. Unlike traditional methods that focus on either extracting good geometric primitives or obtaining proper arrangements of primitives, the emphasis of this method lies in intersecting the primitives (planes only) and seeking for an appropriate combination of them to obtain a manifold polygonal surface model without boundary (Nan and Wonka, 2017). (Nan and Wonka, 2017) shows experiments on point clouds from various sources demonstrate that their method can generate lightweight polygonal surface models of arbitrary piecewise planar objects. Besides, their method is capable of recovering sharp features and is robust to noise, outliers, and missing data.

In this research, automation of 3D polygon geometry from point cloud data through PolyFit was used as a guide. Oriented photos were then marked using the $3 \mathrm{D}$ polygon as a guide for subsequent digitization purposes. The results of this automation are expected to reduce the time and workload for digitizing purposes. Building polygons that have been formed through automation will be to help the modeling of more detailed building facades through manual digitizing on Photomodeler.

\section{PROPOSED METHODOLOGY AND DATA}

\subsection{Overview}

Our approach relies on an algorithm of searching for the best plane among a 3D point cloud, which approximates a model with data features. Figure 1 provides an overview of our procedure. The planning stage includes the selection of study object, planning the photography, selection of devices and establishment of control point and check point. The ground control points (GCPs) and check points (CPs) need to be planned properly before the acquisition of digital photograph. It is necessary to relate measurements derived from the photographic images to a $3 \mathrm{D}$ site coordinate system. The most effective means of achieving this involves placing a number of photogrammetric target points throughout the area of interest. Then, image sequence captured by an unmanned aerial vehicle's camera is carried out on the object.

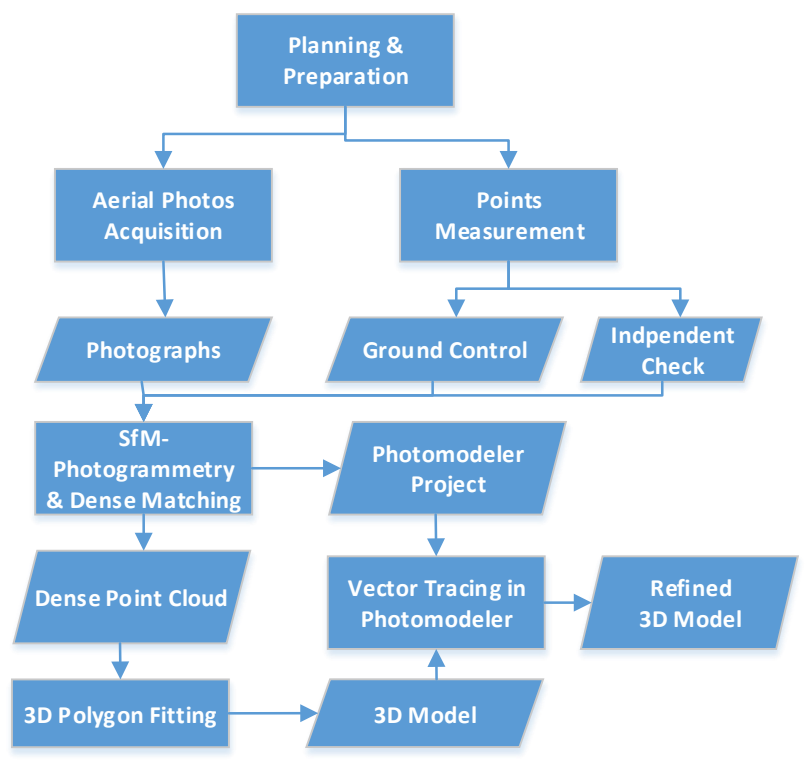

Figure 1. Overall schema of the proposed procedure
Photographs obtained and calibrated were subsequently carried out by the SfM and dense matching process through Photomodeler software to obtain a dense cloud point. The resulting point clouds will then be exported to carry out the next process, namely automation of polygons into $3 \mathrm{D}$ models. The 3D model produced by PolyFit needs to be transformed through Cloud Compare software so that the 3D model can be used as a basis for further digitization. After the 3D model is oriented and in accordance with the initial data, the digitization process can be performed on the details of the building that has not been modeled such as windows, screen walls, and other parts. Digitization is done by using the space intersection principle. This digitization is done to improve details on 3D models (upgrading). We shall now describe in details the various steps.

\subsection{Data Acquisition}

The proposed technique was tested on 2 types of buildings, namely modern architecture and traditional architecture (temples). New building of an office complex in Bandung, named Pondok Hijau Indah Complex, is classified as the modern architecture and one temple in Jogjakarta, named Candi Sari, is classified as the traditional architecture. Figure 2 and Figure 3 show both of the buildings image and point cloud.

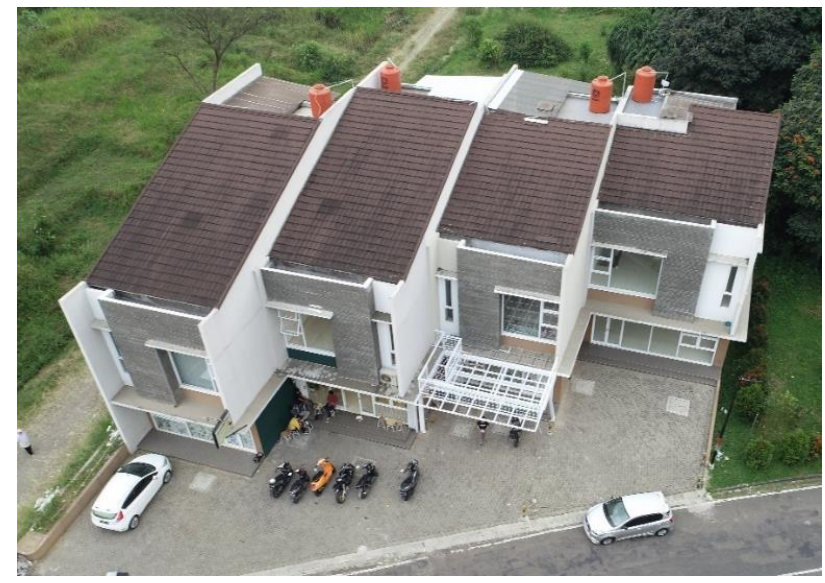

Figure 2. Sample drone photograph of the office Building

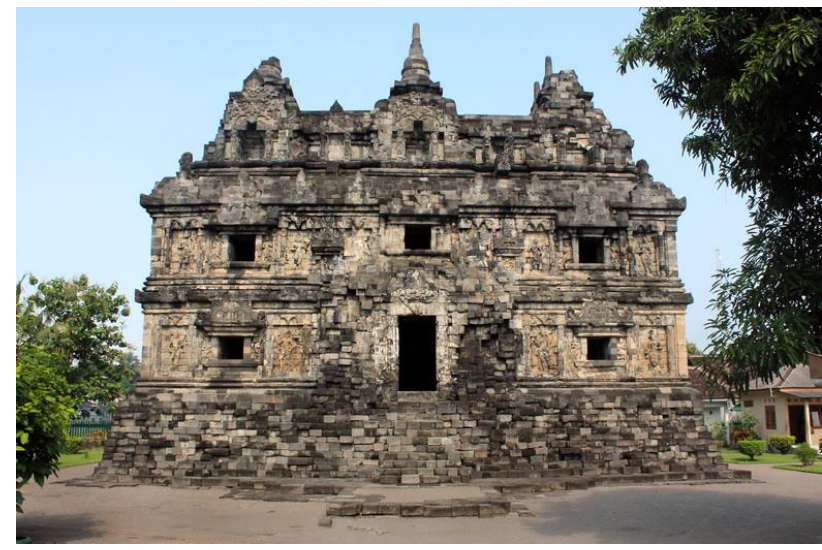

Figure 3. Sample drone photograph of the office Building

In the case of Pondok Hijau Indah Complex, a total of 30 aerial and 19 terrestrial images of the building were captured from the perimeter of the building toward the center, with an image taken about every 10 meters (30 feet). To minimize foreground obstructions in the images and expedite fieldwork, the camera was mounted on a small quadcopter that flew at an elevation of 35 to 45 meters (120 to 150 feet) above the ground. These 
images were combined with images taken at ground level. A total of 18 points were measured using a reflectorless Total Station device; 10 of these points were used as control points for the geo-referencing of the images. The point cloud was generated using the software PhotoModeler. A 3D polygon model of the main building in OBJ format was generated by PolyFit and used as a control/base for drafting directly on the oblique images using Photomodeler. In addition to the OBJ file, a list of all measured points in TXT format was generated. This included the point names, codes, descriptions, coordinates, and their measurement accuracy in all axes. In total, 135 new measurements were made from the images. The measurement accuracy of all points was better than 2 centimeters (5-6 hundredths of a foot) in both position and elevation. In the case of Candi Sari, laser scanning was performed to obtain the interior of the temple while serving as a "bridge" between the interior and the exterior which was acquired using photogrammetry (both terrestrial and UAV). Several carvings inside the temple were also documented using photogrammetry, in order to render them in a higher resolution. The laser scanning data was processed using FARO Scene, while photogrammetric work was done using the software PhotoModeler. A traverse network was established around the temple; with reference points measured using static GNSS. The topographical network was measured in the national cartographic system. The integration of the sensors was performed by using the control points scattered around and inside the temple in the form of coded targets and artificial spheres. These supports were measured using the total stations from at least two stations, thus assuring a spatial intersection for each target. In addition about a dozen detail points were also measured on the front façade of the temple, to serve as check points in the eventual accuracy assessment. The topographical points served as a common reference for both the photogrammetry and laser scanning results, integrating them into the same system automatically after their respective georeferencing steps.

\subsection{SfM-Photogrammetry and Dense Image Matching}

Structure from Motion (SfM) has a fundamental difference from conventional photogrammetry, where in terms of geometry, the position and orientation of the camera are completed automatically without the need to determine targets or ground control points that have been predetermined, which are known to position in 3D. Instead, these parameters are solved simultaneously using repetitive file alignment procedures, based on a feature database that is automatically extracted from a series of overlapping images (Snavely, 2008).

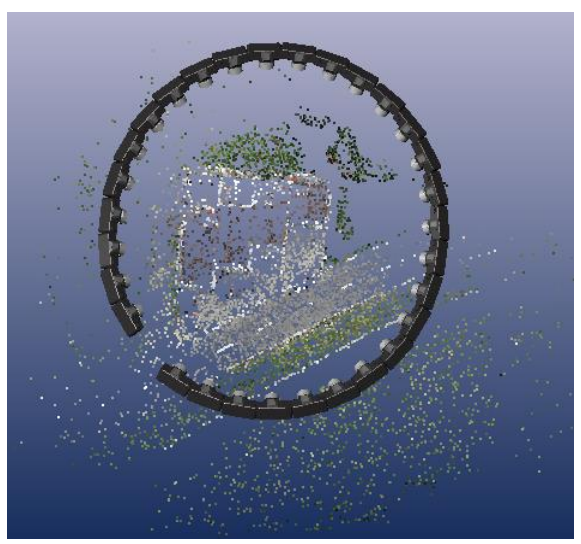

Figure 4. Camera Position and the sparse Point Cloud
SfM-photogrammetry is a solution that integrating SfM and ground control. It is necessary to relate measurements derived from the photographic images to a $3 \mathrm{D}$ site coordinate system. The most effective means of achieving this involves placing a number of photogrammetric target points throughout the area of interest. The sparse point clouds resulting from the SfM process and the camera position distribution can be seen in Figure 4 .

The principle of Structure from Motion is to calculate the position and orientation of the camera that is completed automatically without the need to determine targets or ground control points that have been predetermined, whose positions are known in 3D. These parameters are then solved simultaneously using a repetitive file alignment procedure, based on a feature database that is automatically extracted from a series of overlapping images. The quality of photos oriented in file alignment can be seen in Figure 5.

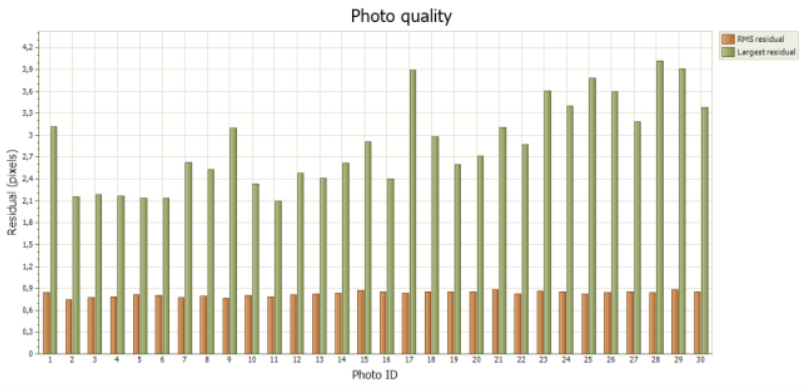

Figure 5. Photogrammetric residual value in pixel (RMS residual and biggest residual)

The quality of photo orientation in the Photomedeler software is a representation of the results of the position of the corresponding object points in each photo due to the alignment process of the file that is influenced by the position and orientation of each camera towards the resulting photo or photos that overlap with the surroundings. After the sparse cloud formed, then the GCP control points were placed at several points scattered in the front area of the building. GCP placement is useful as a photo control so that it is referenced in the UTM coordinate system. The location of GCP points can be seen in Figure 6 while the list of coordinates of GCP control points and their residual values can be seen in Table 1 .

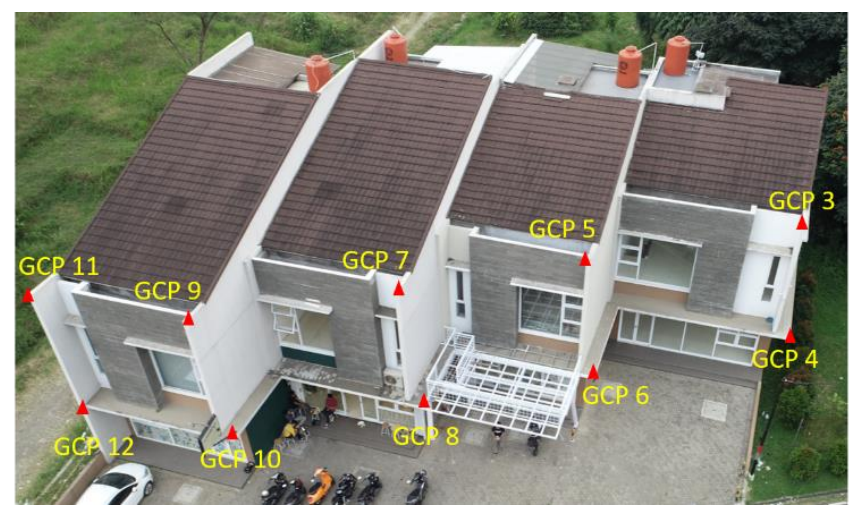

Figure 6. Ground Control Points (GCPs) location distribution

The residual value obtained is the value resulting from GCP measurements in the field of the GCP value after leveling the file in the Photomodeler software. From the ten GCPs, the residual RMS obtained was $0.016 \mathrm{~m}$. 


\begin{tabular}{ccccc}
\hline ID & Easting $(\mathbf{m})$ & Northing $(\mathbf{m})$ & Elev $(\mathbf{m})$ & Residu $(\mathbf{m})$ \\
\hline GCP3 & 786093.385 & 9241501.797 & 947.623 & 0.020 \\
GCP4 & 786093.898 & 9241501.499 & 942.35 & 0.020 \\
GCP5 & 786084.748 & 9241499.688 & 947.623 & 0.014 \\
GCP6 & 786085.385 & 9241499.333 & 942.352 & 0.014 \\
GCP7 & 786078.097 & 9241498.144 & 947.582 & 0.012 \\
GCP8 & 786078.559 & 9241497.849 & 942.376 & 0.014 \\
GCP9 & 786071.228 & 9241496.675 & 947.591 & 0.015 \\
GCP10 & 786071.699 & 9241496.344 & 942.375 & 0.015 \\
GCP11 & 786065.462 & 9241498.405 & 947.629 & 0.019 \\
GCP12 & 786065.656 & 9241498.470 & 942.205 & 0.019 \\
\hline
\end{tabular}

Table 1. Ground Control Points (GCPs) and their value

The check points is needed to check the value of the RMSE (Root Mean Square Error) which states the value of accuracy on the entire result of the photo that has been georeferenced. The check points is measured together with the control points measurement, but this check point has no include on adjusting or shifting the coordinate system. Location of the distribution of the check points can be seen in Figure 7 and the coordinate data measurement and error results can be seen in Table 2 .

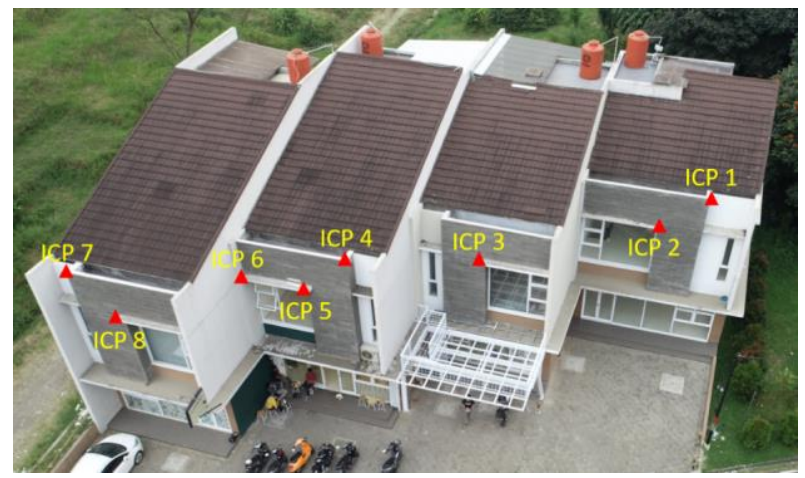

Figure 7. Check Points (ICPs) location distribution

\begin{tabular}{ccccc}
\hline ID & $\begin{array}{c}\text { Easting } \\
(\mathbf{m})\end{array}$ & Northing $(\mathbf{m})$ & $\begin{array}{c}\text { Elevasi } \\
(\mathbf{m})\end{array}$ & $\begin{array}{c}\text { Error } \\
(\mathbf{m})\end{array}$ \\
\hline ICP1 & 786091.698 & 9241502.807 & 947.520 & 0.038 \\
ICP2 & 786089.945 & 9241503.168 & 945.929 & 0.038 \\
ICP3 & 786082.109 & 9241500.913 & 945.986 & 0.153 \\
ICP4 & 786077.013 & 9241498.940 & 947.511 & 0.032 \\
ICP5 & 786075.350 & 9241499.228 & 945.932 & 0.024 \\
ICP6 & 786072.573 & 9241500.176 & 945.938 & 0.024 \\
ICP7 & 786066.811 & 9241498.593 & 947.550 & 0.032 \\
ICP8 & 786068.316 & 9241497.856 & 945.991 & 0.097 \\
\hline
\end{tabular}

Table 2. Check Point (ICPs) and their value

The SmartMatch and DSM (Dense Surface Modeling) tools are used to automatically detect and match features across multiple overlapping photos. The result is a dense point cloud that can either be triangulated/surfaced to form the shape. Projects built with DSM tools can be supplemented with traditional modeling features, such as points and lines.

Whereas Multi-view stereo (MVS) aims to reconstruct a closed 3D model of several photographs calibrated from realistic objects. MVS utilizes a stereo camera that is calibrated using baseline information that is calibrated from a pair of cameras as a geometric constraint based on observation. This method provides distance information throughout the object volume by orbiting the object (Ahmadabadian, 2014). The results of the meeting point cloud development through the MVS method can be seen in Figure 8.

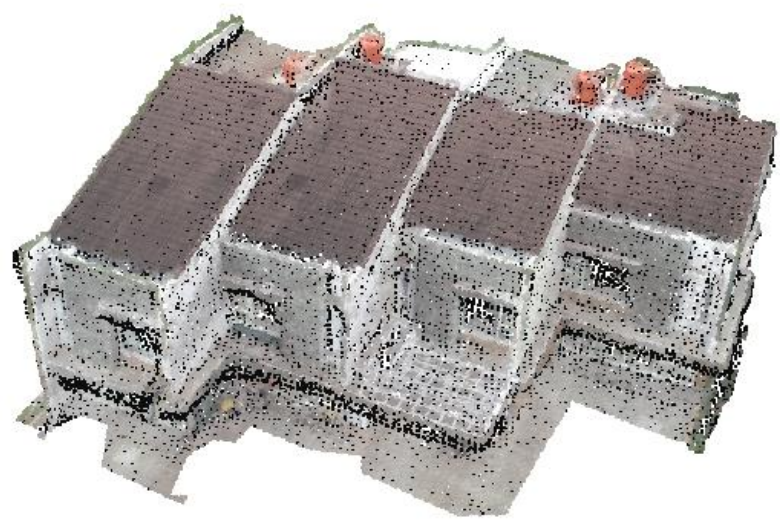

Figure 8. The result of Dense Point Cloud generation with MVS method

\subsection{D Polygon Fitting}

PolyFit is software that offers a new framework for reconstructing lightweight polygon surfaces from cloud points. PolyFit processing is done with the aim to generate 3D models of a building automatically (Nan, 2017). The point cloud from the MVS Photomodeler results is then selected on the part of the building you want to be modeled so that it discards unwanted parts using Cloudcompare or Photomodeler software. After that, normal estimation and primitive extraction is carried out in the Mapple software by entering the appropriate RANSAC parameters to be modeled automatically in PolyFit. The stages of modeling in PolyFit are done automatically.

\subsubsection{Normal Estimation}

Normal estimation is one of the basic stages in 3D point cloud processing. Regression-based normal estimation is first done with the assumption that the surface of an object is smooth (continuous) throughout its surface, and the points around each point on the surface can be approached well by a plane (Zhao et al, 2019). Normal estimation is done to obtain a normal vector at each input data point. The process of determining a vector will be influenced by adjacent points around it through a particular algorithm

\subsubsection{Primitive extraction}

Primitive extraction is carried out through the RANSAC method. The RANSAC method contains three unspecified general parameters (Fischler, 1981): fault tolerance used to determine whether a point fits a model, the number of subsets to try, and the threshold $\mathrm{t}$, which is the number of compatible points used to imply that the correct model has been found. Through Mapple software, primitive extraction is done by entering RANSAC parameters that are suitable for point clouds and building models. The RANSAC parameter values used in primitive extraction on Mapple can be seen in Table 3.

Of the five parameters, the most influential in primitive extraction are the Minimum Support and Distance Threshold. The value of 500 at the minimum support is determined based on the minimum number of points needed to form a surface. If the value is too small, then the number of iterations needed will be more and more and requires a longer processing time. However, if the value is too large it can cause the formation of a primitive as it should. While the distance threshold is 
determined based on the ratio between the distance of adjacent points to the diagonal of the point cloud box space. In practice, the other three parameters do not significantly influence the number entered is the number that has been printed automatically by the Mapple software.

\begin{tabular}{cc}
\hline Parameter RANSAC & Nilai \\
\hline Minimum Support & 500 \\
Distance Threshold & 0.002 \\
Bitmap Resolution & 0.02 \\
Normal Threshold & 0.8 \\
Overlook Probability & 0.001 \\
\hline
\end{tabular}

Table 3. RANSAC parameter value which are used in PolyFit Software

After primitive extraction using the RANSAC method on the 3D point cloud data set, it can be continued as input to the PolyFit software. The stages in PolyFit have four parts, namely Refinement (improvement), Hypothesis (hypothesis), Datafitting (mounting data), and Optimization (optimization).

\subsubsection{Refinement}

Because of the noise and outliers (especially for point clouds that are processed through Multiview Stereo), RANSAC can produce some unwanted planar segments. Unwanted planar segments usually have a random orientation and few points are involved. To overcome this problem, the initial planar segment is iteratively corrected using the field improvement algorithm (Nan, 2017). The refinement process is carried out to select whether there are outliers or surfaces that do not fit the model. If it is an outlier, the data will be discarded. If an inappropriate surface has an angle value below the fault tolerance limit, a merge will be performed using a certain algorithm.

\subsubsection{Hypothesis}

By making stronger assumptions on objects, the researchers further arranged the reconstruction problem and adjusted the combined form (ie a combination of some basic primitives) to a point cloud. In PolyFit, this idea is generalized to reconstruct planar objects piece by piece, and the reconstruction is based on optimization under strict limits that guarantee various polygon surface models (Nan, 2017). The results of improvements and hypotheses can be seen in Figure 9.
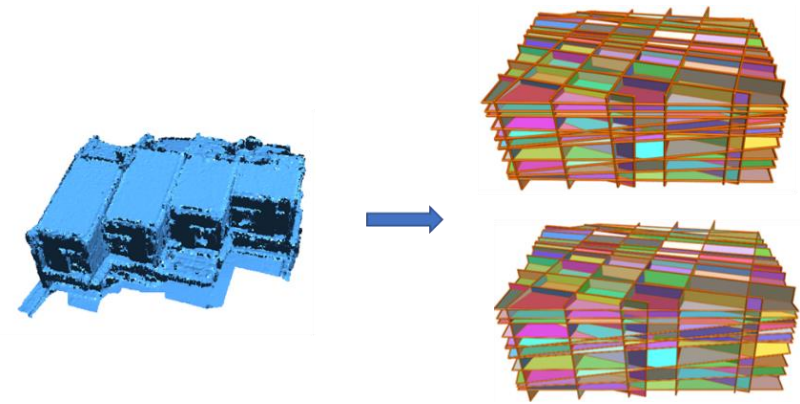

Figure 9. Refinement result and data set hypothesis

\subsubsection{Data-fitting}

Data fitting or data installation is intended to evaluate the quality of mounting a field in a point cloud while also calculating the notation that corresponds to the level of trust. This is defined to measure the percentage of points that did not contribute to the final reconstruction. Intuitively, the term mounting data refers more to the selection of surfaces that are close to the input points and are supported by the same regional points. This term has values in the range of 0 to 1 where a value of 1 indicates noise-free and outlier input data (Nan, 2017).

\subsubsection{Optimization}

This stage is the last stage to reconstruct 3D objects automatically through PolyFit. It can be seen that the fields formed will be intersected with other intersecting fields. This intersection produces various kinds of combinations of surface products that are formed. The problems contained in this stage can be resolved using Gurobi solver (Nan, 2017). The results of optimization can be seen in Figure 10.
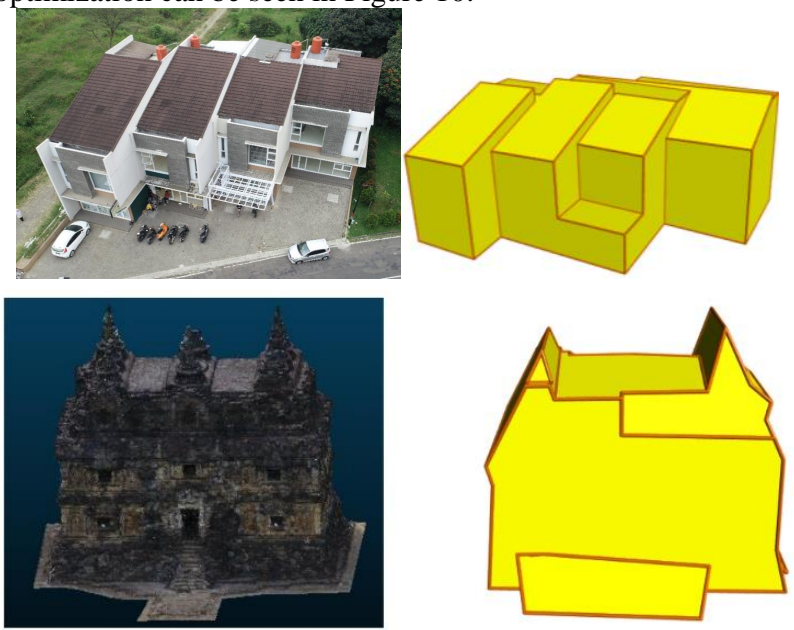

Figure 10. Final result of PolyFit Optimization in front look

\subsection{Detail Digitizing}

A 3D polygon model of the main building in $\mathrm{OBJ}$ format was generated by PolyFit and was used as a control/base for drafting directly on the oblique images using Photomodeler.

The modeling results in PolyFit are still referenced in the local coordinate system. Therefore, it is necessary to transform so that the 3D model can be referenced in a coordinate system, namely UTM Zone $48 \mathrm{~S}$ by using Cloudcompare software. This referenced model can later be used as a base for digitizing the Photomodeler. The transformation parameter values shown in Figure 11 show that the $\mathrm{X}$ translational value $=786066,625, \mathrm{Y}$ translation $=9241495.00$ and $\mathrm{Z}$ translation $=940.594299$ with no rotation and scaling elements of 1.00

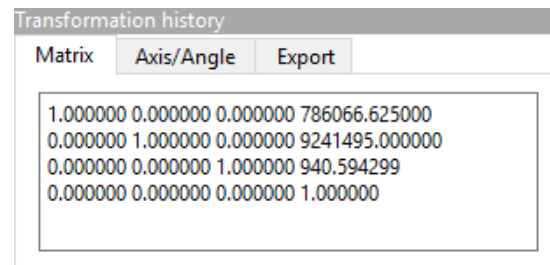

Figure 11. 3D Matrix Transformation in Cloudcompare

Furthermore, importing a 3D model that has been transformed and continued digitizing on the Photomodeler uses the principle of intersection of space where each point of the same object in different photographs is observed so that there are lines that intersect exactly on the object. Objects observed can occur a slight shift in different photos that can be caused by resolution 
and image quality or observer errors. The results of the observed point quality can be seen in Figure 12

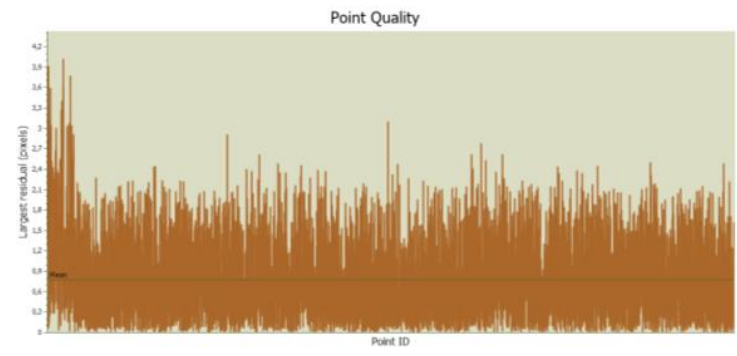

Figure 12. The largest residual value and the average residual per object point observed in pixels

\section{RESULTS AND DISCUSSION}

\subsection{Polygonal surface deformations}

To find out the points distribution that contribute to the formation of the 3D model plane, it is necessary to compare the position of point cloud to the nearest $3 \mathrm{D}$ model plane so that the distance value is obtained. Point cloud distribution deviation values to the 3D model can be seen in the histogram in Figure 14 and its statistical parameters can be seen in Table 4. The minimum distance value is the closest distance formed between the point cloud and the plane.

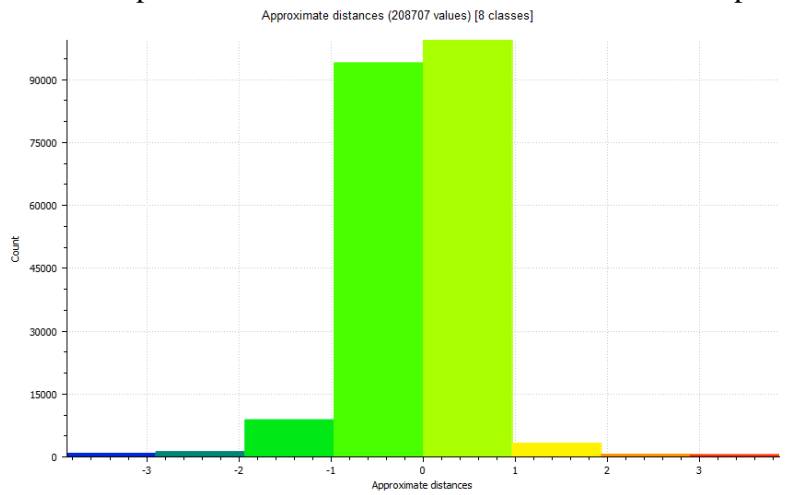

Figure 14. Deviation value histogram of point cloud ( $\mathrm{X}$ axis in meter)

\begin{tabular}{cc}
\hline Parameter & Nilai $(\mathbf{m})$ \\
\hline Min dist. & 0 \\
Max dist. & 3,867 \\
Avg dist. & 0,234 \\
Sigma & 0,470 \\
\hline
\end{tabular}

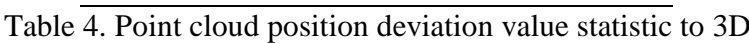
model

Maximum distance states the furthest distance between point cloud and formed field. The average distance is the average distance formed by each point cloud to its nearest plane. Whereas Sigma states the acceptable limit distribution of point cloud deviation.

As it seen statistically where the point cloud is centered on the middle value of the histogram (value 0 ) which has a light green color. While on the left, the negative values are shown by the dark blue. Vice versa, getting to the right shows a positive value that is perceived by red, which means that the point cloud is not right on the plane. Visualization of point cloud distribution can be seen in Figure 15.

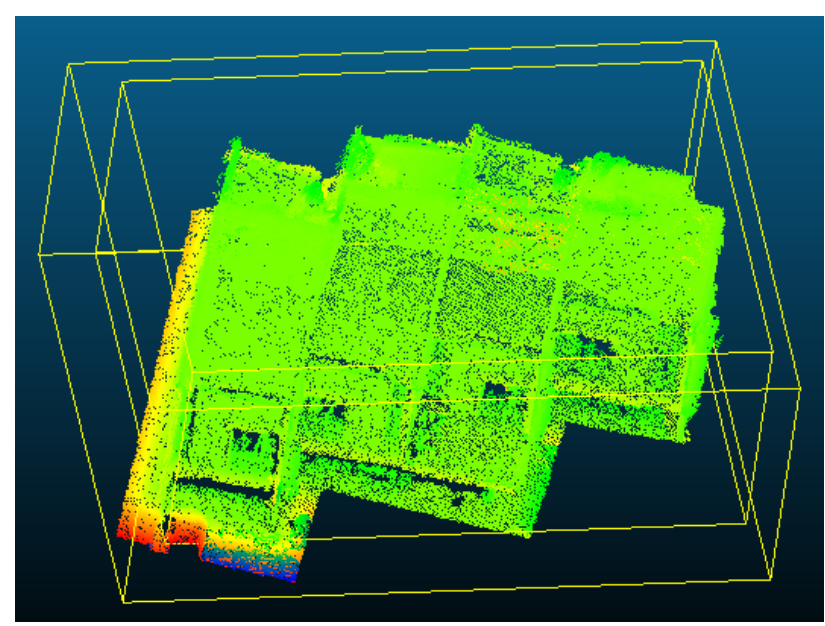

Figure 15. Visualization of the distribution of point cloud intersections against fields

\subsection{Evaluation of monoscopic-derived measurements}

The previous stages have produced 3D models of buildings that are generally modeled on the shape of the building. However, details of buildings such as windows, partition walls, terraces, and others are still not reflected in the model. Therefore, further digitization is needed using Photomodeler on the basis of using 3D models that have been formed in general. Digitizing on a Photomodeler uses the principle of intersection of space where each point of the same object on different photographs is observed so that there are lines that intersect exactly on the object. Objects observed can occur a slight shift in different photos that can be caused by resolution and image quality or observer errors.

To reduce the RMS value at each point observed, more than two photographs can be observed. The more photos involved in the observation, the smaller the RMS value of the observed object residues. The results of digitization on Photomodeler using the basis of the 3D model can be seen in Figure 16.

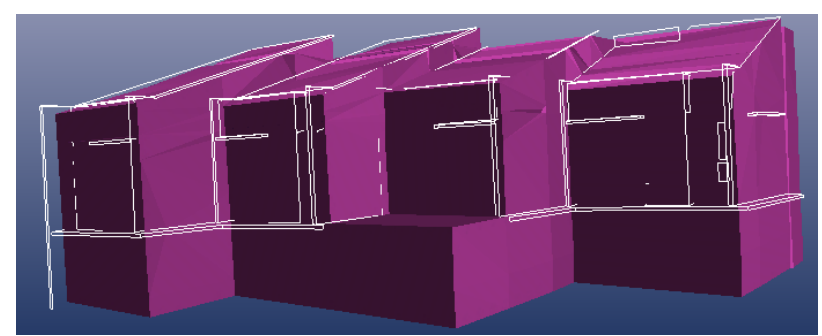

Figure 16. Photomodeler digitization results on the basis of 3D models

The RMSE value of the digitized point is under 3 pixels. While the point that has the highest RMSE value is 2381 which is located at the lower end of the tile at 2.687 pixels. The location of point 2381 can be seen in Figure 17. 


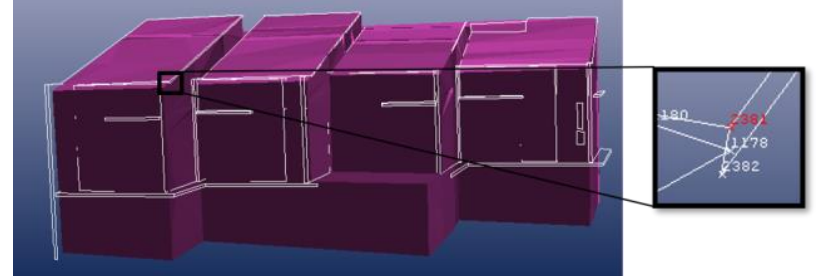

Figure 17. The location of the point that has the highest RMSE value

To find out how suitable the 3D model formed with the photogrammetry project, then the coordinates of the values of the points were considered to be the same either in the 3D model or in the digitization results. The location of the points that are checked can be seen in Figure 18. The results of the difference in the coordinates of the two can be seen in Table 5 .

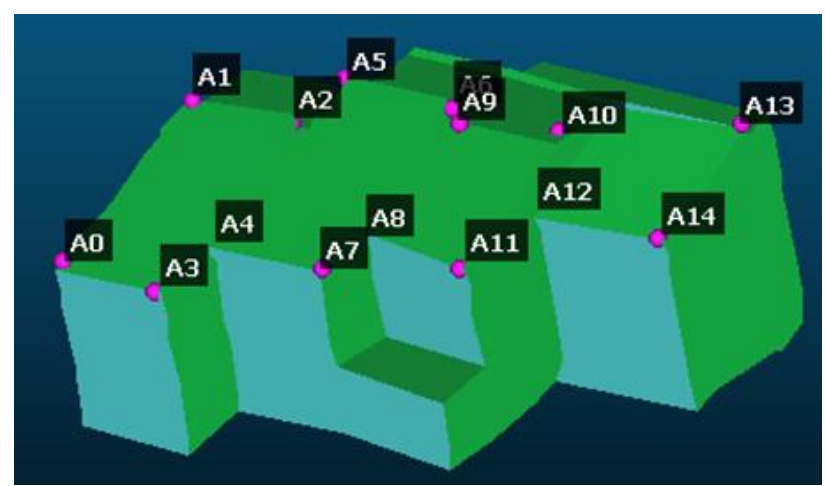

Figure 18. Check point location

\begin{tabular}{ccccc}
\hline ID & $\mathbf{d X}(\mathbf{m})$ & $\mathbf{d Y}(\mathbf{m})$ & $\mathbf{d Z}(\mathbf{m})$ & Total $(\mathbf{m})$ \\
\hline A0 & $-0,077$ & 0,715 & 0,218 & 0,751 \\
A1 & 0,046 & 0,0571 & 0,048 & 0,088 \\
A2 & 0,103 & 0,001 & 0,055 & 0,117 \\
A3 & 0,252 & 0,737 & 0,266 & 0,823 \\
A4 & 0,276 & 0,288 & $-0,013$ & 0,399 \\
A5 & 0,140 & $-0,471$ & 0,159 & 0,516 \\
A6 & $-0,049$ & $-0,433$ & 0,094 & 0,446 \\
A7 & 0,199 & 0,277 & 0,240 & 0,417 \\
A8 & 0,274 & 0,782 & 0,207 & 0,854 \\
A9 & 0,229 & 0,456 & 0,157 & 0,533 \\
A10 & 0,586 & $-0,330$ & 0,282 & 0,729 \\
A11 & 0,445 & 0,859 & 0,182 & 0,984 \\
A12 & 0,286 & 0,264 & 0,168 & 0,424 \\
A13 & 0,274 & $-0,636$ & 0,075 & 0,697 \\
A14 & 0,489 & 0,067 & 0,082 & 0,501 \\
\hline
\end{tabular}

Table 5. Point cloud position deviation value statistic to 3D

\section{CONCLUSIONS}

This research demonstrates that digital close range photogrammetry technique which utilizes a non-metric digital camera at drone is capable of modelling object study.

In the dense cloud construction through the SfM and MVS methods, what needs to be considered is the number and distribution of photos surrounding the research object so that the dense cloud results are obtained with good geometry and dispersion and in accordance with the model. In addition, consideration needs to be given to the use of an appropriate coordinate system for further processing.

3D polygon reconstruction using the PolyFit method is carried out through various stages, namely normal estimation, primitive extraction, refinement, hypothesis, data-fitting, and optimization. In primitive extraction, the RANSAC method is used where each value of each parameter is needed to produce the number of primitives according to the shape of the building. The parameters that are very influential are the minimum support and distance threshold. The input point cloud data is very influential on the results of the $3 \mathrm{D}$ model.

The level of detail of the resulting 3D polygon geometry depends very much on the quality and quantity of the dense cloud resulting from the SfM and MVS photogrammetric processes. From the trial results, it can be seen that the resulting point cloud is not very detailed due to the insufficient number and position / orientation of the photo. This will result in not appearing several digitizing results on the basis of $3 \mathrm{D}$ polygons.

\section{ACKNOWLEDGEMENTS}

The authors would like to express their gratitude to the SAME Perancis 2018 for the funding of the research.

\section{REFERENCES}

Ahmadabadian, Ali Hosseininaveh. (2014). Photogrammetric Multi-View Stereo and Imaging Network Design. Department of Civil, Environmental and Geomatic Engineering University College London (UCL).

Fischler, M. A., dan Bolles, R. C. (1981). Random Sample Consensus: A Paradigm for Model Fitting with Applications to Image Analysis and Automated Cartography. Comm. of the $A C M, \mathrm{Vol} 24$, pp 381-395.

Nan, L., dan Peter Wonka. (2017). PolyFit: Polygonal Surface Reconstruction from Point Clouds. The IEEE International Conference on Computer Vision (ICCV), 2017, pp. 2353-2361.

Snavely, N., Seitz, S.M., \& Szeliski, R. (2008). Modeling the World from Internet Photo Collections. International Journal of Computer Vision November 2008, Volume 80, Issue 2, pp 189210

Zhao, R., Pang, M., Liu, C., dan Zhang, Y. (2019). Robust Normal Estimation for 3D LiDAR Point Clouds in Urban Environments. Sensors 2019, 19, 1248

Barsanti, S.G., Remondino, F., Fenández-Palacios, B.J., Visintini, D., 2014. Critical factors and guidelines for 3D surveying and modelling in Cultural Heritage. Int. J. Herit. Digit. Era 3, 141-158.

Hanke, K., Grusenmeyer, P., 2002. Architectural Photogrammetry: Basic theory,Procedures, Tools, Tutorial of Architectural Photogrammetry, Corfu

Macher, H., Landes, T., Grussenmeyer, P., 2017. From Point Clouds to Building Information Models: 3D Semi-Automatic Reconstruction of Indoors of Existing Buildings. Appl. Sci. 7, $1-30$. 
Murtiyoso, A., Grussenmeyer, P., 2019. Point cloud segmentation and semantic annotation aided by GIS data for heritage complexes, in: The International Archives of the Photogrammetry, Remote Sensing and Spatial Information Sciences. pp. 523-528.

Murtiyoso, A., Grussenmeyer, P., 2017. Documentation of heritage buildings using close-range UAV images: dense matching issues, comparison and case studies. Photogramm. Rec. 32, 206-229.

Nan, L., Wonka, P., 2017. PolyFit: Polygonal Surface Reconstruction from Point Clouds in ICCV. Remondino, F., Rizzi, A., 2010. Reality-based 3D documentation of natural and cultural heritage sites-techniques, problems, and examples. Appl. Geomatics 2, 85-100. 